\title{
Maximizing the Strong Triadic Closure in Split Graphs and Proper Interval Graphs
}

\author{
Athanasios L. Konstantinidis ${ }^{1}$ and Charis Papadopoulos ${ }^{2}$ \\ 1 Department of Mathematics, University of Ioannina, Greece \\ skonstan@cc.uoi.gr \\ 2 Department of Mathematics, University of Ioannina, Greece \\ charis@cs.uoi.gr
}

\begin{abstract}
In social networks the STRONG TRIADIC ClOSURE is an assignment of the edges with strong or weak labels such that any two vertices that have a common neighbor with a strong edge are adjacent. The problem of maximizing the number of strong edges that satisfy the strong triadic closure was recently shown to be NP-complete for general graphs. Here we initiate the study of graph classes for which the problem is solvable. We show that the problem admits a polynomialtime algorithm for two unrelated classes of graphs: proper interval graphs and trivially-perfect graphs. To complement our result, we show that the problem remains NP-complete on split graphs, and consequently also on chordal graphs. Thus we contribute to define the first border between graph classes on which the problem is polynomially solvable and on which it remains NP-complete.
\end{abstract}

1998 ACM Subject Classification F.2 Analysis of Algorithms and Problem Complexity, G.2.2 Graph Theory

Keywords and phrases strong triadic closure, polynomial-time algorithm, NP-completeness, split graphs, proper interval graphs

Digital Object Identifier 10.4230/LIPIcs.ISAAC.2017.53

\section{Introduction}

Predicting the behavior of a network is an important concept in the field of social networks [9]. Understanding the strength and nature of social relationships has found an increasing usefulness in the last years due to the explosive growth of social networks (see e.g., [2]). Towards such a direction the STRONG TRIADIC Closure principle enables us to understand the structural properties of the underlying graph: it is not possible for two individuals to have a strong relationship with a common friend and not know each other [12]. Such a principle stipulates that if two people in a social network have a "strong friend" in common, then there is an increased likelihood that they will become friends themselves at some point in the future. Satisfying the Strong Triadic Closure is to characterize the edges of the underlying graph into weak and strong such that any two vertices that have a strong neighbor in common are adjacent. Since users interact and actively engage in social networks by creating strong relationships, it is natural to consider the MAXSTC problem: maximize the number of strong edges that satisfy the STRONG TRIADIC Closure. The problem has been shown to be NP-complete for general graphs while its dual problem of minimizing the number of weak edges admits a constant factor approximation ratio [28].

In this work we initiate the computational complexity study of the MAXSTC problem in important classes of graphs. If the input graph is a $P_{3}$-free graph (i.e., a graph having

(c) (i) Athanasios L. Konstantinidis and Charis Papadopoulos;

c. licensed under Creative Commons License CC-BY

28th International Symposium on Algorithms and Computation (ISAAC 2017).

Editors: Yoshio Okamoto and Takeshi Tokuyama; Article No. 53; pp. 53:1-53:12

Leibniz International Proceedings in Informatics

LIPICS Schloss Dagstuhl - Leibniz-Zentrum für Informatik, Dagstuhl Publishing, Germany 
no induced path on three vertices which is equivalent with a graph that consists of vertexdisjoint union of cliques) then there is a trivial solution by labeling strong all the edges. Such an observation might falsely lead into a graph modification problem, known as CLUSTER DELETION problem (see e.g., [3, 14]), in which we want to remove the minimum number of edges that correspond to the weak edges, such that the resulting graph does not contain a $P_{3}$ as an induced subgraph. More precisely the obvious reduction would consist in labeling the deleted edges in the instance of Cluster DELETION as weak, and the remaining ones as strong. However, this reduction fails to be correct due to the fact that the graph obtained by deleting the weak edges in an optimal solution of MAXSTC may contain an induced $P_{3}$, so long as those three vertices induce a triangle in the original graph (prior to deleting the weak edges). We stress that there are examples on split graphs (Figure 1) and proper interval graphs (Figure 3) showing such a difference.

To the best of our knowledge, no previous results were known prior to our work when restricting the input graph for the MAXSTC problem. It is not difficult to see that for bipartite graphs the MAXSTC problem has a simple polynomial-time solution by considering a maximum matching that represent the strong edges [15]. In fact such an argument regarding the maximum matching generalizes to the larger class of triangle-free graphs. Also notice that for triangle-free graphs a set of edges is a maximum matching if and only if it is formed by a solution for the Cluster Deletion problem. It is well-known that a maximum matching of a graph corresponds to a maximum independent set of its line graph that represents the adjacencies between the edges [10]. As previously noted, for general graphs it is not necessarily the case that a maximum matching corresponds to the optimal solution for MAXSTC. Here we show a similar characterization for MAXSTC by considering the adjacencies between the edges of a graph that participate in induced $P_{3}$ 's. Such a characterization allows us to exhibit properties towards an optimal solution of MAXSTC.

Due to the nature of the $P_{3}$ existence that enforce the labeling of weak edges, there is an interesting connection to problems related to the square root of a graph; a graph $H$ is a square root of a graph $G$ and $G$ is the square of $H$ if two vertices are adjacent in $G$ whenever they are at distance one or two in $H$. Any graph does not have a square root (for example consider a simple path), but every graph contains a subgraph that has a square root. Although it is NP-complete to determine if a given chordal graph has a square root [21], there are polynomial-time algorithms when the input is restricted to bipartite graphs [20], or proper interval graphs [21], or trivially-perfect graphs [25]. Among several square roots that a graph may have, one can choose the square root with the maximum or minimum number of edges [5, 23]. The relationship between MAXSTC and to that of determining square roots can be seen as follows. In the MAxSTC problem we are given a graph $G$ and we want to select the maximum possible number of edges, at most one from each induced $P_{3}$ in $G$. Thus we need to find the largest subgraph (in terms of the number of its edges) $H$ of $G$ such that the square of $H$ is a subgraph of $G$. However the known results related to square roots were concerned with deciding if the whole graph has a (maximum or minimum) square root and there are no such equivalent formulations related to the largest square root.

Our main motivation is to understand the complexity of the problem on subclasses of chordal graphs, since the class of chordal graphs (i.e., graphs having no chordless cycle of length at least four) finds important applications in both theoretical and practical areas related to social networks $[1,19,26]$. More precisely two famous properties can be found in social networks. For most known social and biological networks their diameter, that is, the length of the longest shortest path between any two vertices of a graph, is known to be a small constant [17]. On the other hand it has been shown that the most prominent social network 

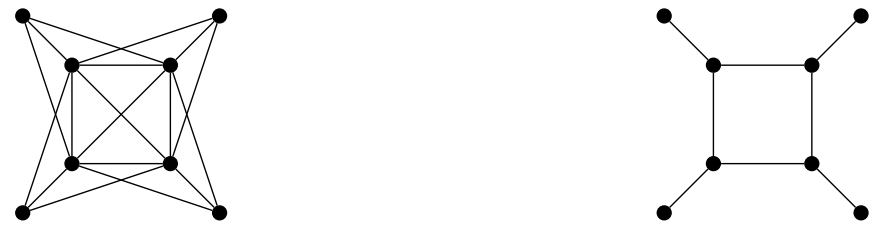

Figure 1 A split graph $G$ is shown to the left side. The right side depicts a solution for MAXSTC on $G$ where the weak edges are exactly the edges of $G$ that are not shown.

subgraphs are cliques, whereas highly infrequent induced subgraphs are cycles of length four [29]. Thus it is evident that subclasses of chordal graphs are close related to such networks, since they have rather small diameter (e.g., split graphs or trivially-perfect graphs) and are characterized by the absence of chordless cycles (e.g., proper interval graphs). Towards such a direction we show that MAXSTC is NP-complete on split graphs and consequently also on chordal graphs. On the positive side, we present the first polynomial-time algorithm for computing MAxSTC on proper interval graphs. Proper interval graphs, also known as unit interval graphs or indifference graphs, form a subclass of interval graphs and they are unrelated to split graphs [27]. By our result they form the first graph class, other than triangle-free graphs, for which MAXSTC is shown to be polynomial time solvable. In order to obtain our algorithm, we take advantage of their clique path (consecutive arrangement of maximal cliques) and apply a dynamic programming on subproblems defined by passing the clique path in its natural ordering. Our structural proofs on proper interval graphs can be seen as useful tools towards settling the complexity of MAXSTC on interval graphs. Furthermore by considering the characterization of the induced $P_{3}$ 's mentioned earlier, we show that MAXSTC admits a simple polynomial-time solution on trivially-perfect graphs (i.e., graphs having no induced $P_{4}$ or $C_{4}$ ).

\section{Preliminaries}

We refer to [4] for our standard graph terminology. Given a graph $G=(V, E)$, a strong-weak labeling on the edges of $G$ is a function $\lambda$ that assigns to each edge of $E(G)$ one of the labels strong or weak; i.e., $\lambda: E(G) \rightarrow$ \{strong, weak $\}$. An edge that is labeled strong (resp., weak) is simply called strong (resp. weak). The strong triadic closure of a graph $G$ is a strong-weak labeling $\lambda$ such that for any two strong edges $\{u, v\}$ and $\{v, w\}$ there is a (weak or strong) edge $\{u, w\}$. In other words, in a strong triadic closure there is no pair of strong edges $\{u, v\}$ and $\{v, w\}$ such that $\{u, w\} \notin E(G)$. The problem of computing a maximum strong triadic closure, denoted by MAXSTC, is to find a strong-weak labeling on the edges of $E(G)$ that satisfies the strong triadic closure and has the maximum number of strong edges. Note that its dual problem asks for the minimum number of weak edges. Here we focus on maximizing the number of strong edges in a strong triadic closure.

Let $G$ be a strong-weak labeled graph. We denote by $\left(E_{S}, E_{W}\right)$ the partition of $E(G)$ into strong edges $E_{S}$ and weak edges $E_{W}$. The graph spanned by $E_{S}$ is the graph $G \backslash E_{W}$. For a vertex $v \in V(G)$ we say that the strong neighbors of $v$ are the other endpoints of the strong edges incident to $v$. We denote by $N_{S}(v) \subseteq N(v)$ the strong neighbors of $v$. Similarly we say that a vertex $u$ is strongly adjacent to $v$ if $u$ is adjacent to $v$ and $\{u, v\}$ is strong.

- Observation 1. Let $G=\left(E_{S}, E_{W}\right)$ be a strong-weak labeled graph. $G$ satisfies the strong triadic closure if and only if for every $P_{3}$ in $G \backslash E_{W}$, the vertices of $P_{3}$ induce a $K_{3}$ in $G$. 


\section{MaxSTC on split graphs}

Here we provide an NP-hardness result for MAxSTC on split graphs. A graph $G=(V, E)$ is a split graph if $V$ can be partitioned into a clique $C$ and an independent set $I$, where $(C, I)$ is called a split partition of $G$. Split graphs form a subclass of the larger and widely known graph class of chordal graphs, which are the graphs that do not contain induced cycles of length 4 or more as induced subgraphs. It is known that split graphs are self-complementary, that is, the complement of a split graph remains a split graph. Hereafter for two vertices $u$ and $v$ we say that $u$ sees $v$ if $\{u, v\} \in E(G)$; otherwise, we say that $u$ misses $v$.

- Lemma 2. Let $G=(V, E)$ be a split graph with a split partition $(C, I)$. Let $E_{S}$ be the set of strong edges in an optimal solution for MAXSTC on $G$ and let $I_{W}$ be the vertices of $I$ that are incident to at least one edge of $E_{S}$.

1. If every vertex of $I_{W}$ misses at least three vertices of $C$ in $G$ then $E_{S}=E(C)$.

2. If every vertex of $I_{W}$ misses exactly one vertex of $C$ in $G$ then $\left|E_{S}\right| \leq|E(C)|+\left\lfloor\frac{\left|I_{W}\right|}{2}\right\rfloor$.

Proof. Let $w_{i}$ be a vertex of $I$ and let $B_{i}$ be the set of vertices in $C$ that are non-adjacent to $w_{i}$. Let $A_{i}$ be the strong neighbors of $w_{i}$ in an optimal solution. For the edges of the clique, there are $\left|A_{i}\right|\left|B_{i}\right|$ weak edges due to the strong triadic closure. Moreover any vertex $w_{j}$ of $I \backslash\left\{w_{i}\right\}$ cannot have a strong neighbor in $A_{i}$. This means that $A_{i} \cap A_{j}=\emptyset$. Notice, however, that both sets $B_{i} \cap B_{j}$ and $A_{i} \cap B_{j}$ are not necessarily empty.

Observe that $I_{W}$ contains the vertices of $I$ that are incident to at least one strong edge. Let $E(A, B)$ be the set of weak edges that have one endpoint in $A_{i}$ and the other endpoint in $B_{i}$, for every $1 \leq i \leq\left|I_{W}\right|$. We show that $2|E(A, B)| \geq \sum_{w_{i} \in I_{W}}\left|A_{i}\right|\left|B_{i}\right|$. Let $\{a, b\} \in E(A, B)$ such that $a \in A_{i}$ and $b \in B_{i}$. Assume that there is a pair $A_{j}, B_{j}$ such that $\{a, b\}$ is an edge between $A_{j}$ and $B_{j}$, for $j \neq i$. Then $a$ cannot belong to $A_{j}$ since $A_{i} \cap A_{j}=\emptyset$. Thus $a \in B_{j}$ and $b \in A_{j}$. Therefore for every edge $\{a, b\} \in E(A, B)$ there are at most two pairs $\left(A_{i}, B_{i}\right)$ and $\left(A_{j}, B_{j}\right)$ for which $a \in A_{i} \cup B_{j}$ and $b \in B_{i} \cup A_{j}$. This means that every edge of $E(A, B)$ is counted at most twice in $\sum_{w_{i} \in I_{W}}\left|A_{i}\right|\left|B_{i}\right|$.

For any two edges $\{u, v\},\{v, z\} \in E(C) \backslash E(A, B)$, observe that they satisfy the strong triadic closure since there is the edge $\{u, z\}$ in $G$. Thus the strong edges of the clique are exactly the set of edges $E(C) \backslash E(A, B)$. In total by counting the number of strong edges between the independent set and the clique, we have $\left|E_{S}\right|=|E(C) \backslash E(A, B)|+\sum_{w_{i} \in I_{W}}\left|A_{i}\right|$. Since $2|E(A, B)| \geq \sum_{w_{i} \in I_{W}}\left|A_{i}\right|\left|B_{i}\right|$, we get

$$
\left|E_{S}\right| \leq|E(C)|+\sum_{w_{i} \in I_{W}}\left|A_{i}\right|\left(1-\left\lfloor\frac{\left|B_{i}\right|}{2}\right\rfloor\right) .
$$

Now the first claim of the lemma holds because $\left|B_{i}\right| \geq 3$ so that $I_{W}=\emptyset$. For the second claim we show that for every vertex of $I_{W},\left|A_{i}\right|=1$. Let $w_{i} \in I_{W}$ such that $\left|A_{i}\right| \geq 2$ and let $B_{i}=\left\{b_{i}\right\}$. Recall that no other vertex of $I_{W}$ has strong neighbors in $A_{i}$. Also note that there is at most one vertex $w_{j}$ in $I_{W}$ that has $b_{i}$ as a strong neighbor. If such a vertex $w_{j}$ exist and for the vertex $b_{j}$ of the clique that misses $w_{j}$ it holds $b_{j} \in A_{i}$, then we let $v=b_{j}$; otherwise we choose $v$ as an arbitrary vertex of $A_{i}$. Observe that no vertex of $I \backslash\left\{w_{i}\right\}$ has a strong neighbor in $A_{i} \backslash\{v\}$ and only $w_{j} \in I_{W}$ is strongly adjacent to $b_{i}$. Then we label weak the $\left|A_{i}\right|-1$ edges between $w_{i}$ and the vertices of $A_{i} \backslash\{v\}$ and we label strong the $\left|A_{i}\right|-1$ edges between $b_{i}$ and the vertices of $A_{i} \backslash\{v\}$. Making strong the edges between $b_{i}$ and the vertices of $A_{i} \backslash\{v\}$ does not violate the strong triadic closure since every vertex of $C \cup\left\{w_{j}\right\}$ is adjacent to every vertex of $A_{i} \backslash\{v\}$. Therefore for every vertex $w_{i} \in I_{W},\left|A_{i}\right|=1$ and by substituting $\left|B_{i}\right|=1$ in the formula for $\left|E_{S}\right|$ we get the claimed bound. 
In order to give the reduction, we introduce the following problem that we call maximum disjoint non-neighborhood: given a split graph $(C, I)$ where every vertex of $I$ misses three vertices from $C$, we want to find the maximum subset $S_{I}$ of $I$ such that the non-neighborhoods of the vertices of $S_{I}$ are pairwise disjoint. In the corresponding decision version, denoted by MaxDisJointNN, we are also given an integer $k$ and the problem asks whether $\left|S_{I}\right| \geq k$. The polynomial-time reduction to MAXDisJoINTNN is given from the classical NP-complete problem 3-Set PACKING [18]: given a universe $\mathcal{U}$ of $n$ elements, a family $\mathcal{F}$ of triplets of $\mathcal{U}$, and an integer $k$, the problem asks for a subfamily $\mathcal{F}^{\prime} \subseteq \mathcal{F}$ with $\left|\mathcal{F}^{\prime}\right| \geq k$ such that all triplets of $\mathcal{F}^{\prime}$ are pairwise disjoint.

- Corollary 3. MAXDisJOINTNN is NP-complete on split graphs.

Now we turn to our original problem MAXSTC. The decision version of MAXSTC takes as input a graph $G$ and an integer $k$ and asks whether there is strong-weak labeling of the edges of $G$ that satisfies the strong triadic closure with at least $k$ strong edges.

- Theorem 4. The decision version of MAXSTC is NP-complete on split graphs.

Proof. Given a strong-weak labeling $\left(E_{S}, E_{W}\right)$ of a split graph $G=(C, I)$, checking whether $\left(E_{S}, E_{W}\right)$ satisfies the strong triadic closure amounts to check in $G \backslash E_{W}$ whether there is a non-edge in $G$ between the endvertices of every $P_{3}$ according to Observation 1 . Thus by listing all $P_{3}$ 's of $G \backslash E_{W}$ the problem belongs to NP. Next we give a polynomial-time reduction to MAXSTC from the MAxDisjointNN problem on split graphs which is NP-complete by Corollary 3. Let $(G, k)$ be an instance of MaxDisjointNN where $G=(C, I)$ is a split graph such that every vertex of the independent set $I$ misses exactly three vertices from the clique $C$. For a vertex $w_{i} \in I$, we denote by $B_{i}$ the set of the three vertices in $C$ that are non-adjacent to $w_{i}$. Let $n=|C|$. We extend $G$ and construct another split graph $G^{\prime}$ as follows (see Figure 2):

- We add $n$ vertices $y_{1}, \ldots, y_{n}$ in the clique that constitutes the set $C_{Y}$.

- We add $n$ vertices $x_{1}, \ldots, x_{n}$ in the independent set that constitutes the set $I_{X}$.

- For every $1 \leq i \leq n, y_{i}$ is adjacent to all vertices of $\left(C \cup C_{Y} \cup I \cup I_{X}\right) \backslash\left\{x_{i}\right\}$.

- For every $1 \leq i \leq n, x_{i}$ is adjacent to all vertices of $\left(C \cup C_{Y}\right) \backslash\left\{y_{i}\right\}$.

Thus $w_{i}$ misses only the vertices of $B_{i}$ from the clique. By construction it is clear that $G^{\prime}$ is a split graph with a split partition $\left(C \cup C_{Y}, I \cup I_{X}\right)$. Notice that the clique $C \cup C_{Y}$ has $2 n$ vertices and $G=G^{\prime}[I \cup C]$.

We claim that $G$ has a solution for MAxDisjointNN of size at least $k$ if and only if $G^{\prime}$ has a strong triadic closure with at least $n(2 n-1)+\left\lfloor\frac{n}{2}\right\rfloor+\left\lceil\frac{k}{2}\right\rceil$ strong edges. Due to space restriction, we only show the one direction.

Assume that $\left\{w_{1}, \ldots, w_{k}\right\} \subseteq I$ is a solution for MAxDisJoInTNN on $G$ of size at least $k$. Since the sets $B_{1}, \ldots, B_{k}$ are pairwise disjoint, there are $k$ distinct vertices $y_{1}, \ldots, y_{k}$ in $C_{Y}$ such that $k \leq n$. We will give a strong-weak labeling for the edges of $G^{\prime}$ that fulfills the strong triadic closure and has at least the claimed number of strong edges. For simplicity, we describe only the strong edges; the edges of $G^{\prime}$ that are not given are all labeled weak. We label the edges between each vertex $w_{i}, y_{i}, x_{i}$ and the three vertices of each set $B_{i}$, for $1 \leq i \leq k$ as follows:

- The edges of the form $\left\{y_{i}, v\right\}$ are labeled strong if $v \in\left(C \cup C_{Y}\right) \backslash B_{i}$ or $v=w_{i}$.

- The edges between $x_{i}$ and the three vertices of $B_{i}$ are labeled strong.

Next we label the edges incident to the rest of the vertices. No edge incident to a vertex of $I \backslash\left\{w_{1}, \ldots, w_{k}\right\}$ is labeled strong. For every vertex $u \in C \backslash\left(B_{1} \cup \cdots \cup B_{k}\right)$ we label the edge $\{u, v\}$ strong if $v \in\left(C \cup C_{Y}\right)$. Let $C_{Y}^{\prime}=\left\{y_{k+1}, \ldots, y_{n}\right\}$ and let $I_{X}^{\prime}=\left\{x_{k+1}, \ldots, x_{n}\right\}$. 


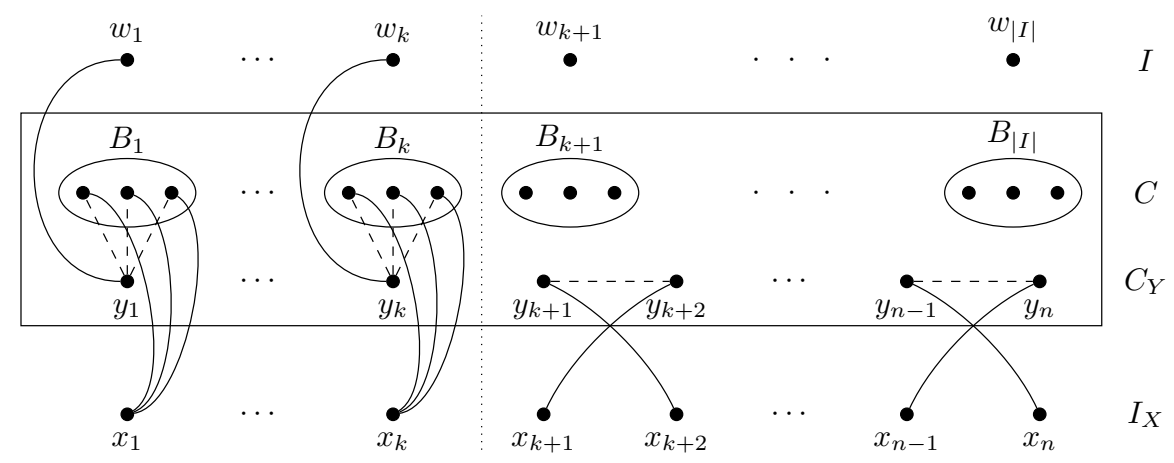

Figure 2 The split graph $\left(C \cup C_{Y}, I \cup I_{X}\right)$ given in the polynomial-time reduction. Every vertex $w_{i}$ misses the vertices of $B_{i}$ and sees the vertices of $\left(C \cup C_{Y}\right) \backslash B_{i}$. Every vertex $x_{i}$ misses $y_{i}$ and sees the vertices of $\left(C \cup C_{Y}\right) \backslash\left\{y_{i}\right\}$. The sets $B_{1}, \ldots, B_{k}$ are pairwise disjoint whereas for every set $B_{j}, k<j \leq|I|$, there is a set $B_{i}, 1 \leq i \leq k$, such that $B_{i} \cap B_{j} \neq \emptyset$. The drawn edges correspond to the strong edges between the independent set and the clique, and the dashed edges are the only weak edges in the clique $C \cup C_{Y}$.

Recall that every vertex $x_{k+j}$ is adjacent to every vertex of $C_{Y}^{\prime} \backslash\left\{y_{k+j}\right\}$. Let $\ell=\left\lfloor\frac{n-k}{2}\right\rfloor$. Let $M=\left\{e_{1}, \ldots, e_{\ell}\right\}$ be a maximal set of pairwise non-adjacent edges in $G^{\prime}\left[C_{Y}^{\prime}\right]$ where $e_{j}=\left\{y_{k+2 j-1}, y_{k+2 j}\right\}$, for $j \in\{1, \ldots, \ell\}$; note that $M$ is a maximal matching of $G^{\prime}\left[C_{Y}^{\prime}\right]$. For every vertex $y \in C_{Y}^{\prime}$, we label the edge $\{y, v\}$ strong if $v \in\left(C \cup C_{Y}\right) \backslash\left\{y^{\prime}\right\}$ such that $\left\{y, y^{\prime}\right\} \in M$. Moreover, for $j \in\{1, \ldots, \ell\}$, the edges $\left\{x_{k+2 j-1}, y_{k+2 j}\right\}$ and $\left\{x_{k+2 j}, y_{k+2 j-1}\right\}$ are labeled strong. Note that if $n-k$ is odd then no edge incident to the unique vertex $y_{n}$ belongs to $M$ and all edges between $y_{n}$ and the vertices of $C \cup C_{Y}$ are labeled strong; in such a case also note that no edge incident to $x_{n}$ is strong.

Let us show that such a labeling fulfills the strong triadic closure. Any labeling for the edges inside $G^{\prime}\left[C \cup C_{Y}\right]$ is satisfied since $G^{\prime}\left[C \cup C_{Y}\right]$ is a clique. Also note that there are no two adjacent strong edges that have a common endpoint in the clique $C \cup C_{Y}$ and the two other endpoints in the independent set $I \cup I_{X}$. If there are two strong edges incident to the same vertex $v$ of the independent set then $v \in\left\{x_{1}, \ldots, x_{k}\right\}$ and $N_{S}[v]=B_{i}$ which is a clique. Assume that there are two adjacent strong edges $\{u, v\}$ and $\{v, z\}$ such that $u \in I \cup I_{X}$, and $v, z \in C \cup C_{Y}$.

- If $u \in\left\{w_{1}, \ldots, w_{k}\right\}$ then $\{u, z\} \in E\left(G^{\prime}\right)$ since every $w_{i}$ misses only the vertices of $B_{i}$.

- If $u \in\left\{x_{1}, \ldots, x_{k}\right\}$ then $v \in B_{i}$ and $\{u, z\} \in E\left(G^{\prime}\right)$ since every vertex $x_{i}$ misses only $y_{i}$.

- If $u \in I_{X} \backslash\left\{x_{1}, \ldots, x_{k}\right\}$ then the strong neighbors of $v$ in $C \cup C_{Y}$ are adjacent to $u$ in $G^{\prime}$ since for the only non-neighbor of $u$ in $C \cup C_{Y}$ there is a weak edge incident to $v$.

Recall that there is no strong edge incident to the vertices of $I \backslash\left\{w_{1}, \ldots, w_{k}\right\}$. Therefore the given strong-weak labeling fulfills the strong triadic closure.

Observe that the number of vertices in $C \cup C_{Y}$ is $2 n$. There are exactly $3 k+\ell$ weak edges in $G^{\prime}\left[C \cup C_{Y}\right]$. Thus the number of strong edges in $G^{\prime}\left[C \cup C_{Y}\right]$ is $n(2 n-1)-3 k-\ell$. There are $k$ strong edges incident to $\left\{w_{1}, \ldots, w_{k}\right\}, 3 k$ strong edges incident to $\left\{x_{1}, \ldots, x_{k}\right\}$, and $2 \ell$ strong edges incident to $I_{X} \backslash\left\{x_{1}, \ldots, x_{k}\right\}$. Thus the total number of strong edges is $n(2 n-1)-3 k-\ell+k+3 k+2 \ell=n(2 n-1)+\ell+k$ and by substituting $\ell=\left\lfloor\frac{n-k}{2}\right\rfloor$ we get the claimed bound. 


\section{Computing MaxSTC on proper interval graphs}

Due to the NP-completeness on split graphs given in Theorem 4, it is natural to consider interval graphs that form another well-studied subclass of chordal graphs. However besides few observations of this section that may be applied for interval graphs, we found several unresolved technicalities. Moreover, to the best of our knowledge, the complexity of the close-related Cluster Deletion problem remains unresolved on interval graphs [3]. Thus we further restrict the input to the class of proper interval graphs that form a proper subclass of interval graphs. Our polynomial solution for MAXSTC on proper interval graphs can be seen as a first step towards determining its complexity on interval graphs.

A graph is a proper interval graph if there is a bijection between its vertices and a family of closed intervals of the real line such that two vertices are adjacent if and only if the two corresponding intervals overlap and no interval is properly contained in another interval. A vertex ordering $\sigma$ is a linear arrangement $\sigma=\left\langle v_{1}, \ldots, v_{n}\right\rangle$ of the vertices of $G$. For a vertex pair $x, y$ we write $x \preceq y$ if $x=v_{i}$ and $y=v_{j}$ for some indices $i \leq j$; if $x \neq y$ which implies $i<j$ then we write $x \prec y$. The first position in $\sigma$ will be referred to as the left end of $\sigma$, and the last position as the right end. We will use the expressions to the left of, to the right of, leftmost, and rightmost accordingly.

A vertex ordering $\sigma$ for $G$ is called a proper interval ordering if for every vertex triple $x, y, z$ of $G$ with $x \prec y \prec z,\{x, z\} \in E(G)$ implies $\{x, y\},\{y, z\} \in E(G)$. Proper interval graphs are characterized as the graphs that admit such orderings, that is, a graph is a proper interval graph if and only if it has a proper interval ordering [24]. We only consider this vertex ordering characterization for proper interval graphs. Moreover it can be decided in linear time whether a given graph is a proper interval graph, and if so, a proper interval ordering can be generated in linear time [24]. It is clear that a vertex ordering $\sigma$ for $G$ is a proper interval ordering if and only if the reverse of $\sigma$ is a proper interval ordering. Two adjacent vertices $u$ and $v$ are called twins if $N[u]=N[v]$. A connected proper interval graph without twin vertices has a unique proper interval ordering $\sigma$ up to reversal $[8,16]$. Figure 3 shows a proper interval graph with its proper interval ordering.

Let us turn our attention to the MAXSTC problem. Instead of maximizing the strong edges of the original graph $G$, we will look at the maximum independent set of the following graph that we call the line-incompatibility graph $\widehat{G}$ of $G$ : for every edge of $G$ there is a node in $\widehat{G}$ and two nodes of $\widehat{G}$ are adjacent if and only if the vertices of the corresponding edges induce a $P_{3}$ in $G$. In a different context the notion of line-incompatibility has already been considered under the term Gallai graph in [22] or as an auxiliary graph in [5]. Note that the line-incompatibility graph of $G$ is a subgraph of the line graph ${ }^{1}$ of $G$. Moreover observe that for a graph $G$, its line graph and its line-incompatibility graph coincide if and only if $G$ is a triangle-free graph.

- Proposition 5. A subset $S$ of edges $E(G)$ is an optimal solution for MAXSTC of $G$ if and only if $S$ is a maximum independent set of $\widehat{G}$.

Therefore we seek for the optimal solution of $G$ by looking at a solution for a maximum independent set of $\widehat{G}$. As a byproduct, if we are interested in minimizing the number of weak edges then we ask for the minimum vertex cover of $\widehat{G}$. We denote by $I_{\widehat{G}}$ the maximum independent set of $\widehat{G}$. To distinguish the vertices of $\widehat{G}$ with those of $G$ we refer to the

\footnotetext{
1 The line graph of $G$ is the graph having the edges of $G$ as vertices and two vertices of the line graph are
} adjacent if and only if the two original edges are incident in $G$. 


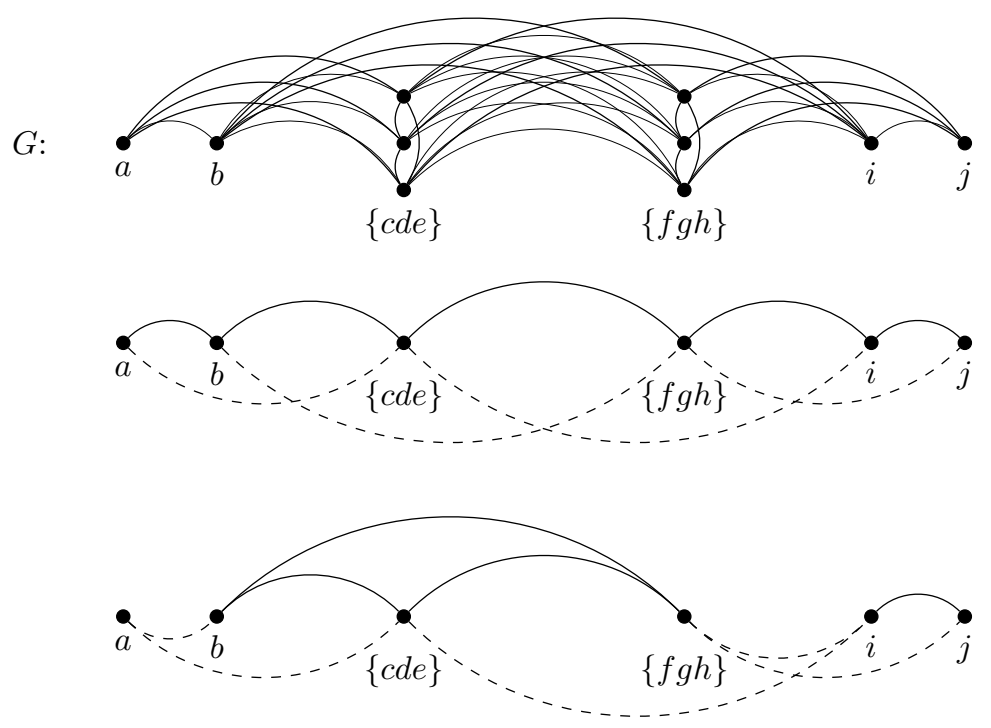

Figure 3 A proper interval graph $G$ and its proper interval ordering. The vertices $\{c, d, e\}$ and $\{f, g, h\}$ form twin sets in $G$. The two lower orderings depict two solutions for MAxSTC on $G$. A solid edge corresponds to a strong edge, whereas a dashed edge corresponds to a weak edge. Observe that the upper solution contains larger number of strong edges than the lower one. Also note that the lower solution consists an optimal solution for the Cluster DeLeTion problem on $G$.

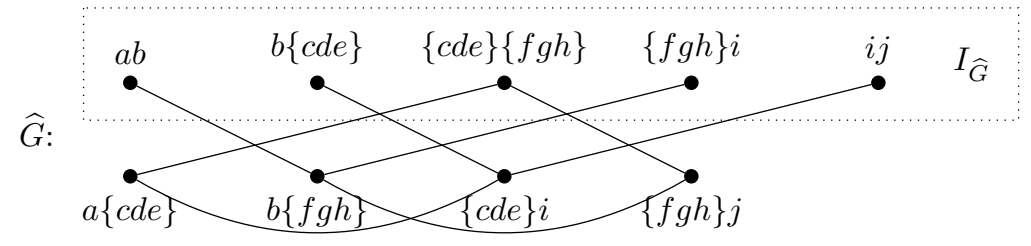

Figure 4 The line-incompatibility graph $\widehat{G}$ of the proper interval graph $G$ given in Figure 3 . The set $I_{\widehat{G}}$ is a maximum weighted independent set of $\widehat{G}$, by taking into account the weight of each node (i.e., an edge of $G$ ) that corresponds to the number of the twin vertices of its endpoints in $G$ (see Lemma 6).

former as nodes and to the latter as vertices. For an edge $\{u, v\}$ of $G$ we denote by $u v$ the corresponding node of $\widehat{G}$. Figure 4 shows the line-incompatibility graph of the proper interval graph given in Figure 3 .

A natural contraction for several graph problems is to group twin vertices since they play the same role on the given graph. With the next result, we show that this is indeed the case for the MAXSTC problem.

- Lemma 6. Let $x$ and $y$ be twin vertices of a graph $G$. Then there is an optimal solution $I_{\widehat{G}}$ such that $x y \in I_{\widehat{G}}$ and for every vertex $u \in N(x), x u \in I_{\widehat{G}}$ if and only if $y u \in I_{\widehat{G}}$.

Lemma 6 suggests to consider a graph $G$ that has no twin vertices as follows. We partition $V(G)$ into sets of twins. For every twin set $W_{x}$ we choose an arbitrary vertex $x$ and remove all its twin vertices except $x$ from $G$. Let $G^{\prime}$ be the resulting graph that has no twin vertices. For every edge $\{x, y\}$ of $G^{\prime}$ we assign a weight equal to the product $\left|W_{x}\right| \cdot\left|W_{y}\right|$. This value corresponds to all edges of the original graph $G$ between the vertices of $W_{x}$ and $W_{y}$. The line-incompatibility graph $\widehat{G^{\prime}}$ of $G^{\prime}$ is constructed as defined above with the only difference that a node of $\widehat{G^{\prime}}$ has weight equal to the weight of its corresponding edge in $G^{\prime}$. Let $I_{G^{\prime}}$ be 
a maximum weighted independent set, that is an independent set of $\widehat{G^{\prime}}$ such that the sum of the weights of its nodes is maximized. Then by Lemma 6 we have $I_{\widehat{G}}=I_{\widehat{G}^{\prime}} \cup S(W)$ where $S(W)$ contains $\left|W_{x}\right|\left(\left|W_{x}\right|-1\right) / 2$ nodes for every twin set $W_{x}$. Therefore we are interested in computing a maximum weighted independent set of $\widehat{G^{\prime}}$. Also note that $G^{\prime}$ is an induced subgraph of the original graph $G$. In order to avoid heavier notation we refer to $\widehat{G^{\prime}}$ as $\widehat{G}$ by assuming that $G$ has no twin vertices and every vertex of $G$ has a positive weight.

Before reaching the details of our algorithm for proper interval graphs, let us highlight the difference between the optimal solution for MAXSTC and the optimal solution for the Cluster Deletion. As already explained in the Introduction a solution for Cluster DELETION satisfies the strong triadic closure, though the converse is not necessarily true. In fact such an observation carries out for the class of proper interval graphs as shown in the example given in Figure 3. For the Cluster Deletion problem twin vertices can be grouped together following a similar characterization with Lemma 6, as proved in [3]. This means that the $P_{3}$-free graph depicted in the lower part of Figure 3 that is obtained by removing its weak edges (i.e., the dashed drawn lines) is an optimal solution for CLUSTER DELETION problem on the given proper interval graph. Therefore when restricted to proper interval graphs the optimal solution for CLuster DELETION does not necessarily imply an optimal solution for MAXSTC.

Let $G$ be a proper interval graph and let $\sigma$ be a proper interval ordering for $G$. We say that a solution $I_{\widehat{G}}$ has the consecutive strong property with respect to $\sigma$ if for any three vertices $x, y, z$ of $G$ with $x \prec y \prec z$ the following holds: $x z \in I_{\widehat{G}}$ implies $x y, y z \in I_{\widehat{G}}$. Our task is to show that such an optimal ordering exists. We start by characterizing the optimal solution $I_{\widehat{G}}$ with respect to the proper interval ordering $\sigma$.

- Lemma 7. Let $x, y, z$ be three vertices of a proper interval graph $G$ such that $x \prec y \prec z$. If $x z \in I_{\widehat{G}}$ then $x y \in I_{\widehat{G}}$ or $y z \in I_{\widehat{G}}$.

Proof. We show that at least one of $x y$ or $y z$ belongs to $I_{\widehat{G}}$. Assume towards a contradiction that neither $x y$ nor $y z$ belong to $I_{\widehat{G}}$. Consider the node $x y$ in $\widehat{G}$. If $x y$ is adjacent to a node $x x_{\ell} \in I_{\widehat{G}}$ then $\left\{x_{\ell}, y\right\} \notin E(G)$. Then observe that $x_{\ell} \prec y$ because $x \prec y$ and $\left\{x_{\ell}, y\right\} \notin E(G)$. Since both $x x_{\ell}$ and $x z$ belong to $I_{\widehat{G}},\left\{x_{\ell}, z\right\} \in E(G)$. This however contradicts the proper interval ordering because $x_{\ell} \prec y \prec z,\left\{x_{\ell}, z\right\} \in E(G)$ and $y$ is non-adjacent to $x_{\ell}$. Thus $x y$ is non-adjacent to any node $x x_{\ell} \in I_{\widehat{G}}$ and, in analogous fashion, $y z$ is non-adjacent to any node $z z_{r} \in I_{\widehat{G}}$.

Now assume that $x y$ is adjacent to a node $y y_{r} \in I_{\widehat{G}}$ and $y z$ is adjacent to a node $y_{\ell} y \in I_{\widehat{G}}$. This means that $\left\{x, y_{r}\right\} \notin E(G)$ and $\left\{z, y_{\ell}\right\} \notin E(G)$. Since $\{x, z\} \in E(G)$, by the proper interval ordering we have $y_{\ell} \prec x \prec y \prec z \prec y_{r}$. Then notice that $\left\{y_{\ell}, y_{r}\right\} \in E(G)$, because both $y y_{r}, y y_{\ell} \in I_{\widehat{G}}$. By the proper interval ordering we know that both $x$ and $z$ are adjacent to $y_{\ell}, y_{r}$, leading to a contradiction to the assumptions $\left\{x, y_{r}\right\} \notin E(G)$ and $\left\{z, y_{\ell}\right\} \notin E(G)$. Therefore at least one of $x y$ or $y z$ belongs to $I_{\widehat{G}}$.

Thus by Lemma 7 we have two symmetric cases to consider. The next characterization suggests that there is a fourth vertex with important properties in each corresponding case.

- Lemma 8. Let $x, y, z$ be three vertices of a proper interval graph $G$ such that $x \prec y \prec z$ and $x z \in I_{\widehat{G}}$.

- If $x y \notin I_{\widehat{G}}$ and $y z \in I_{\widehat{G}}$ then $x y$ is non-adjacent to any node $x_{\ell} x \in I_{\widehat{G}}$ and there $i s$ a vertex $w$ such that $y w \in I_{\widehat{G}},\{x, w\} \notin E(G)$, and $z \prec w$.

- If $x y \in I_{\widehat{G}}$ and $y z \notin I_{\widehat{G}}$ then $y z$ is non-adjacent to any node $z z_{r} \in I_{\widehat{G}}$ and there is a vertex $w$ such that $w y \in I_{\widehat{G}},\{w, z\} \notin E(G)$ and $w \prec x$. 
Now we are ready to show that there is an optimal solution that has the described properties with respect to the given proper interval ordering.

- Lemma 9. There exists an optimal solution $I_{\widehat{G}}$ that has the consecutive strong property with respect to $\sigma$.

Lemma 9 suggests to find an optimal solution that has the consecutive strong property with respect to $\sigma$. In fact by Proposition 5 and the proper interval ordering, this reduces to computing the largest proper interval subgraph $H$ of $G$ such that the vertices of every $P_{3}$ of $H$ induce a clique in $G$.

Let $G$ be a proper interval graph and let $\sigma=\left\langle v_{1}, \ldots, v_{n}\right\rangle$ be its proper interval ordering. For a vertex $v_{i}$ we denote by $\ell(i)$ and $r(i)$ the positions of its leftmost and rightmost neighbors, respectively, in $\sigma$. Observe that for any two vertices $v_{i} \prec v_{j}$ in $\sigma, v_{\ell(i)} \preceq v_{\ell(j)}$ and $v_{r(i)} \preceq v_{r(j)}$ [8]. For $1 \leq j \leq r(1)$, let $V_{j}=\left\{v_{1}, \ldots, v_{j}\right\}$, that is, $V_{j}$ contains the first $j$ vertices in $\sigma$. Observe that any subset of vertices of $V_{j}$ induces a clique in $G$. For the set $V_{j}$ we denote by $B\left(V_{j}\right)$ the value that corresponds to the total weight of the edges incident to $v_{1}$ and each of $v_{2}, \ldots, v_{j}$.

Let $A(G)$ be the value of an optimal solution $I_{\widehat{G}}$ for $G$. For technical reasons we assume that $v_{i} v_{i}$ is an edge of $G$ with weight equal to zero. For every vertex $v_{i}$ we denote by $L[i]=i$ and $R[i]=r(i)$. The vectors $L$ and $R$ are called the rightmost limits of the vertices. Let $A(G, L, R)$ be the value of the optimal solution $I(G, L, R)$ such that for every vertex $v_{i}$ its rightmost strong neighbor $v_{k}$ lies between the positions $L[i]$ and $R[i]$. That is, for every vertex $v_{i}$ with $v_{i} v_{k} \in I(G, L, R)$ and $k$ as large as possible, $L[i] \leq k \leq R[i]$ holds. The key idea is that we try all positions $j$ among the rightmost limits of the first vertex $v_{1}$. This is achieved through the consecutive strong property by making $v_{1}$ strongly adjacent to every vertex of $V_{j}$. Then, however, we need to update accordingly the rightmost limits of each vertex of $V_{j}$ in order to obey the consecutive strong property. As a trivial case observe that if $G$ contains exactly one vertex then $A(G)=0$.

- Lemma 10. Let $G$ be a proper interval graph and let $L$ and $R$ be the rightmost limits of the vertices with respect to $\sigma$. Then $A(G)=A(G, L, R)$ and

$$
A(G, L, R)=\max _{L[1] \leq j \leq R[1]}\left\{A\left(G-\left\{v_{1}\right\}, L_{j}, R_{j}\right)+B\left(V_{j}\right)\right\},
$$

where $L_{j}[i]=\left\{\begin{array}{ll}j & \text { if } i \leq j, \\ L[i] & \text { otherwise }\end{array} \quad\right.$ and $\quad R_{j}[i]= \begin{cases}\min \{r(1), R[i]\} & \text { if } i \leq j, \\ R[i] & \text { otherwise. }\end{cases}$

Now we are equipped with our necessary tools in order to obtain our main result, namely a polynomial-time algorithm that solves the MAXSTC problem on proper interval graphs.

- Theorem 11. There is a polynomial-time algorithm that computes the MAXSTC of a proper interval graph.

\section{$5 \quad$ Concluding remarks}

Given the first study with positive and negative results for the MAXSTC problem on restricted input, there are some interesting open problems. As we pointed out MAXSTC is more difficult than Cluster DELETION in the following sense: a solution for Cluster DELETION forms a solution for MAXSTC but the converse is not necessarily true. We have given examples showing that such an observation carries out for split graphs as well as for 
proper interval graphs. Despite the structural difference of both problems, our result on split graphs points out an important and interesting complexity difference between the two problems: on split graphs CLuSTER DELETION has already been shown to be polynomially solvable [3] whereas we prove that MAXSTC remains NP-complete. It is interesting to explore other graph classes that exhibit the same behavior. Towards such a direction observe that every problem expressible in monadic second order logic (MSOL) with quantification over the vertices and vertex sets can be solved in linear time for graphs of bounded treewidth [7]. Indeed, MAXSTC can be formulated in MSOL: (i) the edges are partitioned into two subsets $E_{S}, E_{W}$ (i.e., a strong-weak labeling), (ii) the endpoints of every path of length two spanned by the edges of $E_{S}$ have an edge (i.e., satisfy the strong triadic closure), and (iii) $\left|E_{S}\right|$ is as large as possible. Therefore there is a linear-time algorithm for MAXSTC on graphs of bounded treewidth [7].

Apart from the structural properties that we proved for the solution on proper interval graphs, the complexity of MAXSTC on interval graphs is still open. Moreover it is natural to characterize the graphs for which their line-incompatibility graph is perfect. Such a characterization will lead to further polynomial cases of MAXSTC, since the problem of finding a maximum independent set of perfect graphs admits a polynomial solution [13]. A typical example is the class of bipartite graphs for which their line graph coincides with their line-incompatibility graph and it is known that the line graph of a bipartite graph is perfect (see for e.g., [4]). As we show next, another paradigm of this type is the class of trivially-perfect graphs.

A graph $G$ is called trivially-perfect (also known as quasi-threshold) if for each induced subgraph $H$ of $G$, the number of maximal cliques of $H$ is equal to the maximum size of an independent set of $H$. It is known that the class of trivially-perfect graphs coincides with the class of $\left(P_{4}, C_{4}\right)$-free graphs, that is every trivially-perfect graph has no induced $P_{4}$ or $C_{4}$ [11]. A cograph is a graph without an induced $P_{4}$, that is a cograph is a $P_{4}$-free graph. Hence trivially-perfect graphs form a subclass of cographs.

- Theorem 12. The line-incompatibility graph of a trivially-perfect graph is a cograph.

By Theorem 12 and the fact that the maximum independent set of a cograph can be computed in linear time [6], MAXSTC can be solved in polynomial time on trivially-perfect graphs. We would like to note that the line-incompatibility graph of a cograph or a proper interval graph is not necessarily a perfect graph.

More general there are extensions and variations of the MAxSTC problem that are interesting to consider as proposed in [28]. An interesting and realistic problem is to allow multiple types of strong edges $S_{0}, S_{1}, \ldots, S_{k}$ that do not allow violating "ordered" $P_{3}$ 's. More precisely the objective is to partition the edges of $G$ into $S_{0}, S_{1}, \ldots, S_{k}$ with $k \geq 1$ so that there is no pair of edges $\{u, v\} \in S_{i}$ and $\{v, w\} \in S_{i}$ such that $\{u, w\} \notin E(G)$ and $\left|S_{1}\right|+\cdots+\left|S_{k}\right|$ is as large as possible.

\section{References}

1 A. B. Adcock, B. D. Sullivan, and M. W. Mahoney. Tree decompositions and social graphs. Internet Mathematics, 12:315-361, 2016.

2 L. Backstrom and J. Kleinberg. Romantic partnerships and the dispersion of social ties: a network analysis of relationship status on facebook. In Proceedings of CSCW 2014, pages 831-841, 2014.

3 F. Bonomo, G. Durán, and M. Valencia-Pabon. Complexity of the cluster deletion problem on subclasses of chordal graphs. Theoretical Computer Science, 600:59-69, 2015. 
4 A. Brandstädt, V. B. Le, and J. Spinrad. Graph Classes: A Survey. Society for Industrial and Applied Mathematics, 1999.

5 M. Cochefert, J.-F. Couturier, P. A. Golovach, D. Kratsch, and D. Paulusma. Parameterized algorithms for finding square roots. Algorithmica, 74:602-629, 2016.

6 D.G. Corneil, H. Lerchs, and L.K. Stewart. Complement reducible graphs. Discrete Applied Mathematics, 3:163-174, 1981.

7 B. Courcelle. The monadic second-order logic of graphs i: Recognizable sets of finite graphs. Information and Computation, 85:12-75, 1990.

8 X. Deng, P. Hell, and J. Huang. Linear-time representation algorithms for proper circulararc graphs and proper interval graphs. SIAM J. Comput., 25:390-403, 1996.

9 D. Easley and J. Kleinberg. Networks, Crowds, and Markets: Reasoning About a Highly Connected World. Cambridge University Press, 2010.

10 J. Edmonds. Paths, trees and flowers. Canad. J. Math., 17:449-467, 1965.

11 M.C. Golumbic. Trivially perfect graphs. Discrete Mathematics, 24:105-107, 1978.

12 M. Granovetter. The strength of weak ties. American J. of Sociology, 78:1360-1380, 1973.

13 M. Grötschel. Polynomial algorithms for perfect graphs. North-Holland Mathematics Studies, 21:325-356, 1984.

14 P. Heggernes, D. Lokshtanov, J. Nederlof, C. Paul, and J. A. Telle. Generalized graph clustering: recognizing $(p, q)$-cluster graphs. In Proceedings of WG 2010, pages 171-183, 2010.

15 J. E. Hopcroft and R. M. Karp. An $n^{5 / 2}$ algorithm for maximum matchings in bipartite graphs. SIAM J. Comput., 2:225-231, 1973.

16 L. Ibarra. The clique-separator graph for chordal graphs. Discrete Applied Mathematics, 157:1737-1749, 2009.

17 M. O. Jackson. Social and economic networks. Princeton University press, vol. 3, 2008.

18 R. M. Karp. Reducibility among combinatorial problems. Complexity of Computer Computations, pages 85-103, 1972.

19 D. J. Kleitman and R. V. Vohra. Computing the bandwidth of interval graphs. SIAM J. Disc. Math., 3:373-375, 1990.

20 L. C. Lau. Bipartite roots of graphs. ACM Transactions on Algorithms, 2:178-208, 2006.

21 L. C. Lau and D. G. Corneil. Recognizing powers of proper interval, split, and chordal graphs. SIAM J. Disc. Math., 18:83-102, 2004.

22 V. B. Le. Gallai graphs and anti-gallai graphs. Discrete Mathematics, 159:179-189, 1996.

23 V. B. Le, A. Oversberg, and O. Schaudt. Polynomial time recognition of squares of ptolemaic graphs and 3-sun-free split graphs. Theoretical Computer Science, 602:39-49, 2015.

24 P. J. Looges and S. Olariu. Optimal greedy algorithms for indifference graphs. Computers G3 Mathematics with Applications, 25:15-25, 1993.

25 M. Milanič and O. Schaudt. Computing square roots of trivially perfect and threshold graphs. Discrete Applied Mathematics, 161:1538-1545, 2013.

26 J. L. Pfaltz. Chordless cycles in networks. In Proceedings of ICDE Workshops 2013, pages 223-228, 2013.

27 F. S. Roberts. Indifference graphs. In Proof Techniques in Graph Theory, Academic Press, New York, pages 139-146, 1969.

28 S. Sintos and P. Tsaparas. Using strong triadic closure to characterize ties in social networks. In Proceedings of KDD 2014, pages 1466-1475, 2014.

29 J. Ugander, L. Backstrom, and J. Kleinberg. Subgraph frequencies: Mapping the empirical and extremal geography of large graph collections. In Proceedings of $W W W 2013$, pages 1307-1318, 2013. 